\title{
FLUJO DECAJA Y TASA DE CORTE PARA LA EVALUACIÓN DE PROYECTOS DE INVERSIÓN
}

\section{RESUMEN \\ La evaluación de un proyecto se realiza a través de diferentes métodos. EI VAN (Valor Actual Neto) y la TIR (Tasa Interna de Retorno) se encuentran entre los más utilizados, siendo el flujo de cajay la tasa de corte los parámetrosmínimos paralaaplicación de estos métodos. En el presente trabajo, pretendemos esclarecer cuál debería ser la tasa de corte adecuada para el flujo de caja, tomando como base el criterio del aportante de capital. A nuestro entender, la teoría respectiva aún no ha tratado este tema con profundidad. \\ Palabras Clave: Flujo de caja,tasa de corte, VAN,TIR.}

Cashflow ANDDiscountrate FOR THE EVALUATION OF INVESTMENTPROJECTS ABSTRACT

Projects are assessed through different methods. The VNA and the TIR are the mos used, being the crucial factors the cash flow and the discount rate. This article is aimed at making it clear what discount rate should be appropriate, taking into account the fund contributor criterion. We think that this subject has not been treated in depth by the respective theory.

Key words: CashFlow, discount rate, VNA TIR.

(1) Magíster en Administración y Finanzas. Profesor del Departamento de Gestión y Producción Industrial, UNMSM. E-mail: danielmavila@yahoo.es

(2) Licenciado en Ciencias Contables. Profesor de la Facultad de Ciencias Contables, UNMSM. E-mail: epolaf@viabcp.com

\section{INTRODUCCIÓN}

La mayoría de los métodos, que evalúan una alternativa de inversión, utilizan el flujo de caja para hacer la estimación respectiva, flujo que, en la literatura especializada, aparece bajo distintos nombres y usos, los que precisaremos en el presente trabajo.

El Valor Actual Neto (VAN) y la Tasa Interna de Retorno (TIR) tienen como fuente de cálculo y de comparación una tasa de corte, que puede ser: EI Costo Promedio Ponderado de Capital (CPPK), la Tasa Mínima Atractiva de Retorno (TMAR), Costo de oportunidad del Capital(CoK), etc., tasas que, en muchos casos, se emplean indistintamente para hallar el Valor Actual Neto Económico (VANE), el Valor Actual Neto Financiero (VANF), la Tasa Interna de Retorno Económico (TIRE) y la Tasa Interna de Retorno Financiero (TIRF), por lo que creemos necesario precisar algunos conceptos para lograr una adecuada toma de decisiones.

\section{PREMISAS}

Cuando se elabora el flujo de caja, se debe partir de los siguientes supuestos:

a. Presunción de los flujos. Estos pueden establecerse en condiciones de certidumbre, cuando tenemos plena certeza de su ocurrencia. Las otras variantes, respecto a la certeza de los flujos aparecen en condiciones de riesgo, cuando asignamos probabilidades de ocurrencia para cada uno de los elementos que conforman el flujo. Cuando es imposible establecer probabilidades de ocurrencia, se puede recurrir a técnicas de investigación operativa en la que, además de los modelos matemáticos, intervienen criterios subjetivos por parte de los agentes que participan en la toma de decisiones, esto sobre la base de experiencias, pálpitos, etc., calificándose este escenario como de incertidumbre.

b. Confiables. En el sentido en que los proyectistas no manipulan las cifras en beneficio personal o por quedar bien ante algún prestamista, inversionista, etc.

c. Moneda constante. Lo cual supone que no se diseñará un proyecto asumiendo un proceso inflacionario. Sin embargo, para los planes de desembolso del financiamiento, considerando que usualmente las tasas monetarias del sistema financiero toman en cuenta la inflación, éstos tendrán que deflactarse. Para ello se tendrá que establecer una hipótesis sobre la tasa de inflación. Otra alternativa sería tomar, como 
referencia, la tasa que proyecta el Banco Central de Reserva del Perú (BCRP).

Si el financiamiento fuera en moneda extranjera, habría que considerar las tasas de devaluación de la moneda del préstamo, con respecto a la moneda con la cual se está elaborando el flujo.

\section{d. Los cálculos se establecerán después de la} deducción del impuesto a la renta de tercera categoría. En consecuencia, la aplicación de la tasa de corte también será posterior al impuesto, al tener en cuenta el escudo fiscal, producto del endeudamiento con terceros.

e. Se estimará un valor de salvamento, tanto para los activos fijos como para el capital de trabajo, rubros que establecen la diferencia entre un flujo de caja -considerado para efectos de evaluación de una alternativa de inversión del presupuesto- y el flujo de caja contable de una empresa en marcha.

f. Una única actividad empresarial. Si la empresa promotora es una empresa en marcha y se encuentra evaluando el proyecto, los estados financieros (EEFF) tendrían que formularse de manera consolidada, ya que podrían aparecer tasas impositivas marginales y/o variaciones, esto debido a la depreciación de los activos [6].

\section{FLUJO DE CAJA PARA LA EVALUACIÓN DE PROYECTOS}

Precisemos las distintas denominaciones que la literatura especializada le da al flujo que se empleará para el cálculo de los indicadores económicos financieros de un proyecto.

Arlette Beltrán et al. (2001) [2] indican que el estado de flujos de efectivo muestra el efecto de los cambios o variaciones de dinero en efectivo y/o equivalentes de efectivo en un periodo determinado. De manera alternativa, los autores nos hacen una reseña del flujo de fondos, al que han denominado fuentes y usos del efectivo, que servirá como base para los cálculos, en tanto y en cuanto todos los flujos proyectados contemplen que las ventas y las compras, la participación laboral, los tributos, etc., se efectúen al contado o se paguen en el momento de su determinación. De no cumplirse esta premisa, el flujo de fondos no servirá para la evaluación, debido a que los métodos como el VAN y la TIR consideran que el dinero tiene un valor cronológico.

Apaza (2003) [1] señala que el "cash flow" es un instrumento contable que muestra el dinero que genera la empresa, tanto a través de sus actividades ordinarias como extraordinarias. El término "cash flow" es una contracción de "flow of cash", cuya traducción sería "flujo de caja", definido como el flujo interno de fuentes y usos del dinero durante un periodo determinado. El mismo autor señala que el flujo de caja y el presupuesto del efectivo son los listados de ingresos y egresos de efectivo que se esperan se produzcan, tratándolos en consecuencia como términos sinónimos.

Para Collazos(1999) [3], uno de los criterios para la elaboración de un flujo es clasificarlo en tres tipos de actividades: De operación, de inversión y de financiamiento. Este criterio permite identificar la capacidad del proyecto para generar flujos de entrada de efectivo netos, a partir de las operaciones que dejen el remanente suficiente para pagar deudas, intereses y dividendos, así como identificar los requerimientos de financiamiento y los efectos que éstos tendrán sobre el efectivo generado por las transacciones de financiamiento e inversión.

Según Park (1997) [4], el flujo de efectivo representa las ganancias futuras generadas por el uso productivo del activo fijo, los gastos de capital y los gastos periódicos, tales como: Salarios, materia prima, costos operativos, costos de mantenimiento, impuestos, etc.

Van Horne et al. (1994) [5] señalan que el estado de flujos de fondos, también conocido como estado de fuente y aplicación de fondos o como estado de los cambios en la posición financiera, ayuda para evaluar el uso que la empresa hace de los fondos y para llevar a cabo el financiamiento de estos empleos de fondos.

En nuestro país, desde el año 2000, el estado de flujo de efectivo ha reemplazado al estado de cambios en la situación financiera, para efectos de presentación de los estados financieros de una empresa en marcha, siendo el propósito de este flujo el de reportar los flujos de entrada y salida de efectivo de la cuenta Caja Bancos, desagregados en tres categorías: Actividades de operación, de financiamiento y de inversión. Al utilizarse con otros estados financieros y reportes, este estado ayuda a evaluar la capacidad de una empresa para generar efectivo por dividendos e inversiones y enfatiza las diferencias entre el ingreso neto y el egreso neto de efectivo, además de identificar las necesidades de financiamiento externo. Si bien sirve de ayuda para analizar recepciones y desembolsos de efectivo, tiene una deficiencia importante: Al centrarse en las transacciones de efectivo, se omiten transacciones importantes que no son en efectivo, que corresponden al período en curso. Por consiguiente, si queremos 
entender en su plenitud las transacciones de operación, inversión y financiamiento, nos convendría preparar un estado de flujo de fondos.

El presupuesto del efectivo es indispensable para que el gerente financiero determine las necesidades de efectivo que una empresa tenga a corto plazo y, de acuerdo con ello, planear su financiamiento a corto plazo. Cuando la elaboración del presupuesto del efectivo se amplía con la finalidad de incluir una escala de posibles resultados, el gerente financiero puede evaluar el riesgo del negocio y la liquidez de la empresa, planeando un margen de seguridad realista. Como podrá deducirse, este estado financiero difiere grandemente con el flujo de efectivo, por lo que no se aplica para efectos de evaluación de un proyecto.

\section{EVALUACIÓN DE PROYECTOS}

La evaluación se compone de métodos que permiten medir la viabilidad del futuro negocio y nos servirá para decidir si es conveniente o no asignar recursos para la consecución de los objetivos trazados. Es el principal objetivo de los estudios de preinversión, los cuales comprenden las etapas de: estudios preliminares (perfil), la prefactibilidad (anteproyecto preliminar) o la factibilidad. Desde el punto de vista comercial o empresarial, debemos distinguir los fines de la evaluación para determinar la rentabilidad económica, financiera y la del accionista, asimismo tomaremos como sinónimos la tasa de corte con la tasa de descuento.

\section{Rentabilidad económica}

Mediante los indicadores VANE y TIRE, se evalúa la bondad de un proyecto para generar recursos que permitan demostrar la viabilidad de éste, independientemente de la estructura financiera. Como contrapartida, no se considerarán amortizaciones financieras ni gastos financieros.

Muchos proyectistas y autores de tratados de evaluación de proyectos elaboran el flujo de caja, ya sea para proyectos con financiamiento o sin él y nos muestran, en todos los casos, el cálculo del VAN y de la TIR, sin precisar si se trata de una evaluación económica (VANE, TIRE) o financiera (VANF, TIRF), detalle que, a nuestro entender, resulta indispensable para efectuar una adecuada toma de decisiones. Otra discrepancia que tenemos con diversos tratadistas se refiere a la tasa de corte que se emplea para el cálculo del VANE o para la comparación con la TIRE. Estos estudiosos indican que la tasa de corte a utilizar es la que representa la tasa de rentabilidad de la empresa o la que el(los) accionista(s) desea(n) obtener. Sin embargo, consideramos que la tasa de corte debería ser aquella que satisfaga a todos los agentes que están financiando el proyecto; en otras palabras, el CPPC (Costo Promedio Ponderado de Capital). El proyecto será intrínsecamente bueno, en la medida que cumpla con la obtención de la rentabilidad mínima establecida y la tasa de interés del pasivo, exigidas por los aportantes del capital de inversión.

\section{Rentabilidad financiera}

Si elaboramos el flujo de caja, tomando en cuenta el financiamiento y la subsecuente amortización y pago de intereses, estaremos hablando del flujo de caja financiero (FCF), el cual nos permitirá obtener el VANF y el TIRF, que nos servirá, a su vez, para determinar la conveniencia de ejecutar el proyecto considerando, como tasa de corte, la tasa de rentabilidad que la empresa promotora desee. Además, al compararlos con los indicadores VANE o TIRE, estaremos determinando si el financiamiento es conveniente o no.

\section{Rentabilidad del inversionista (accionista) de un proyecto}

El estado de resultados o estado de ganancias y pérdidas (EEGGPP) nos muestra la utilidad que se ha calculado, tomando en consideración ciertas normas contables, las que, sin embargo, no nos permiten conocer la disponibilidad efectiva del dinero, mientras que al inversionista le interesará conocer las cantidades efectivas de dinero que podría retirar del proyecto en evaluación, esto sin alterar el funcionamiento del negocio. Las cantidades que se retiren estarán en función a la política de dividendos que la futura empresa adopte, tanto en dividendos en efectivo como en dividendos en acciones liberadas.

\section{CASO PRÁCTICO}

La empresa Obladi S.A. está analizando un proyecto para la fabricación de penetrómetros de bolsillo. Se cuenta con la información que se muestra en el Cuadro 1, donde las tasas están dadas en términos anuales y los montos en unidades monetarias.

De la comparación de los escenarios sin endeudamiento (VANE) y con endeudamiento (VANF) se observa que, desde el punto de vista del accionista, resultaría mejor que la empresa no contrate pasivos; sin embargo, esta decisión dependerá de los recursos propios que posea la empresa promotora.

Desde el punto de vista de la empresa, resulta más conveniente el endeudamiento porque genera un mayor valor de la empresa, el cual se verá reflejado, entre otros parámetros, por el valor de salvamento. 


\section{Cuadro 1. Información de la empresa Obladi S.A.}

\begin{tabular}{|lc}
\hline Descripción & Valores \\
Ventas en los años 1, 2 y 3 000 ; 000 y 000 \\
Costos fijos operativos, sin considerar depreciación & 4000 \\
\hline Costo de oportunidad de la empresa (CoK) & $18 \%$ \\
\hline Tasa mínima atractiva del(os) accionista(s) (TMAR) & $16 \%$ \\
Política de reparto anual de dividendos en efectivo & $10 \%$ \\
Costo efectivo cobrado por el prestamista & $15 \%$ \\
\hline Plan de reembolso, cuotas de amortización & 2 \\
constante & $13 \%$ \\
\hline CPPK después de impuestos & 80 000 \\
Inversión en activos fijos & $10 \%$ \\
Depreciación & $75 \%$ del valor en libros \\
\hline Valor de salvamento & 3 años \\
\hline Horizonte de proyección & $7 / 3$ \\
Relación Deuda/Capital para cubrir la inversión & $65 \%$ \\
Margen de ganancia variable & $30 \%$ \\
\hline Impuesto a la renta & $10 \%$ \\
\hline Participación laboral & \\
\hline
\end{tabular}

\section{Cuadro 2. Escenario sin financiamiento}

\section{Estado de ganancias y pérdidas (sin endeudamiento)}

\begin{tabular}{|c|c|c|c|}
\hline Años & 1 & 2 & 3 \\
\hline$=$ Utilidad Neta ( sin considerar deuda) & 8820 & 21105 & 20475 \\
\hline - Dividendos en efectivo (10\%) & 882 & 2111 & 2048 \\
\hline$=$ Utilidades retenidas & 7938| & 26933 & 45360 \\
\hline
\end{tabular}

Flujo de caja económico

\begin{tabular}{|c|c|c|c|c|}
\hline Años & 0 & 1 & 2 & 3 \\
\hline Flujo de caja económico & -80000 & 16820 & 29105 & 84475 \\
\hline Flujo de caja econ. del & -24000 & 882 & 2111 & \\
\hline
\end{tabular}

$\left(^{*}\right)$ Incluye el valor de salvamento y las utilidades retenidas.

\section{Cuadro 3. Escenario con financiamiento}

Estado de ganancias y pérdidas (con financiamiento)

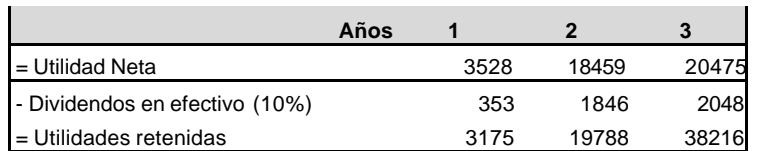

\section{Flujo de caja financiero}

\begin{tabular}{|c|c|c|c|}
\hline Años & 0 & 2 & 3 \\
\hline Flujo de caja financiero & $-24000-16472$ & -1541 & 84475 \\
\hline Flujo de caja financiero del acci & -24000 & 1846 & 82263 * \\
\hline
\end{tabular}

$\left.{ }^{*}\right)$ Incluye el valor de salvamento y las utilidades retenidas.
Cálculos de valor actual neto económico **

\begin{tabular}{cc|c|c|} 
VANE & $\begin{array}{c}\text { Tasa de } \\
\text { descuento }\end{array}$ & Criterio & Punto de vista \\
16748 & $13 \%$ & CPPK & Proyecto per se \\
6571 & $18 \%$ & CoK & Empresa \\
\hline 35608 & $16 \%$ & TMAR & Inversionistas \\
\hline
\end{tabular}

$\left.{ }^{(*}\right)$ Nuestra propuesta es que para hallar el VANE debemos tomar como tasa de corte el CPPK.

\section{Cálculos de valor actual neto financiero **}

\begin{tabular}{|c|c|c|c|}
\hline VANF & $\begin{array}{c}\text { Tasa de } \\
\text { descuento }\end{array}$ & Criterio & Punto de vista \\
\hline 19114 & $13 \%$ & CPPK & $\begin{array}{l}\text { Aportantes de } \\
\text { capital }\end{array}$ \\
\hline 12348 & $18 \%$ & CoK & Empresa \\
\hline 30379 & $16 \%$ & TMAR & Inversionistas \\
\hline
\end{tabular}

(**) Nuestra propuesta es que para hallar el VANF debemos tomar como tasa de corte el CoK. 
La comparación efectuada es estática. Para un análisis más detallado de los efectos de la tasa de descuento, habría que recurrir a técnicas de simulación.

\section{CONCLUSIONES}

Para hallar el VANE deberíamos tomar como tasa de corte el Costo Promedio Ponderado de Capital y para encontrar el VANF deberíamos tomar como tasa de corte el Costo de Oportunidad del Capital de los aportantes del capital propio.

La rentabilidad de cada accionista de la futura empresa estará en función de la política de dividendos que se adopte y de la tasa mínima de retorno que establezca cada aportante del capital social.

El flujo de fondos, también conocido como fuentes y usos del efectivo, se utiliza como base para los cálculos de los indicadores económicos financieros, en tanto y en cuanto los flujos proyectados contemplen que las ventas y las compras, la participación laboral, los tributos, etc., se efectúen al contado o se paguen en el momento de su respectivo cálculo. De no cumplirse esta premisa, el flujo de fondos no serviría para los efectos de la evaluación de un proyecto.

El flujo de caja financiero y los indicadores VANF y TIRF se compararán con otras alternativas de financiamiento, donde no sólo el costo del crédito será el parámetro decisivo, sino también la liquidez que pueden proporcionar las alternativas de financiamiento.

Un análisis de flujo de fondos, entendido como flujo de efectivo, puede resultar limitante. Un análisis del flujo de fondos, en el que los fondos se definan estrictamente como "efectivo" pueden resultar críticos para una completa evaluación del negocio. Las compras y las ventas a crédito, la adquisición de propie- dad a cambio de acciones o bonos, el intercambio de una parte de la propiedad por otra, el disponer de una parte o del total del terreno o la edificación de la propia empresa son sólo algunos ejemplos de las transacciones que no se reportarían en un estado del flujo de fondos, sobrevalorando, en este caso, la rentabilidad del proyecto.

La comparación de los indicadores económicos y financieros se ha efectuado en condiciones de certidumbre. Para un análisis más detallado de los efectos de la tasa de descuento, habría que recurrir a las técnicas de análisis de la sensibilidad, de análisis de los escenarios o de técnicas de simulación.

\section{BIBLIOGRAFÍA}

1. Apaza, Mario. (2003). Revista Entre Líneas. Año 14. №209 Diciembre II, 2003. p. 58-66.

2. Beltrán, A. y Cueva, H. (2003). Evaluación Privada de Proyectos. $2^{\mathrm{a}}$ ed. Ed. Universidad del Pacífico. p. 293-334.

3. Collazos, J. (1999). Inversión y Financiamiento de Proyectos. $1^{\mathrm{a}}$ ed. Editorial San Marcos. Lima, Perú. p. 196-214.

4. Park, Chan S. (1997). Ingeniería Económica Contemporánea. Addison Wesley lberoamericana. p. 196-199.

5. Van Horne, J. C. y Wachowicz, J. M. (1994). Fundamentos de Administración Financiera. 8ª ed. Prentice-Hall Hispanoamericana. México. p. 207214.

6. Velásquez, A. (2000). Proyectos de Inversión. Cómo Hacer Estudios de Factibilidad de Proyectos y Negocios. 1aㅡ ed. Editora Impresora Amarilys EIRL. Lima, Perú. p. 301-304. 